\title{
Design study of beam position monitors for measuring second-order moments of charged particle beams
}

\author{
Kenichi Yanagida, ${ }^{*}$ Shinsuke Suzuki, and Hirofumi Hanaki \\ Japan Synchrotron Radiation Research Institute, Accelerator Division, Accelerator Group I, \\ 1-1 Kouto 1 chome, Sayo-cho, Sayo-gun, Hyogo, 679-5198, Japan
}

(Received 21 April 2011; published 13 January 2012)

\begin{abstract}
This paper presents a theoretical investigation on the multipole moments of charged particle beams in two-dimensional polar coordinates. The theoretical description of multipole moments is based on a single-particle system that is expanded to a multiparticle system by superposition, i.e., summing over all single-particle results. This paper also presents an analysis and design method for a beam position monitor (BPM) that detects higher-order (multipole) moments of a charged particle beam. To calculate the electric fields, a numerical analysis based on the finite difference method was created and carried out. Validity of the numerical analysis was proven by comparing the numerical with the analytical results for a BPM with circular cross section. Six-electrode BPMs with circular and elliptical cross sections were designed for the SPring-8 linac. The results of the numerical calculations show that the second-order moment can be detected for beam sizes $\geqq 420 \mu \mathrm{m}$ (circular) and $\geqq 550 \mu \mathrm{m}$ (elliptical).
\end{abstract}

DOI: 10.1103/PhysRevSTAB.15.012801

PACS numbers: 29.20. $-c$

\section{INTRODUCTION}

Today, beam position monitors (BPMs) are not only used in ring accelerators but also in linear accelerators (linacs) for essential beam diagnostics purposes. Typically a symmetric arrangement of four pickup electrodes serves as a BPM detector to obtain the position of the beam centroid (beam position). Recently, a BPM with eight electrodes has been developed and tested to obtain the second-order moments of the beam charge distribution $[1,2]$ in a beam duct with circular cross section.

To design a BPM pickup, an analytical or numerical calculation of the electric field is essential. In the case of a four-electrode BPM, this is rather simple, because the beam can be treated as a point charge. In the case of a BPM with more electrodes, as used for the measurement of higher-order moments, the field calculation must be performed considering the beam charge distribution in the two-dimensional transverse plane.

Traditional two-dimensional $n$ th-order charge moments were obtained by convolution integrals of a charge distribution $\rho(x, y)$ with an extraction function $x^{j} y^{n-j}[2,3]$ in Cartesian coordinates. This calculation yields the independent moments in general, but requires extensive algebra.

Because there are only two independent elements of two-dimensional $n$ th-order moments, we propose two moments that are orthogonal in the polar coordinates. Consequently, the $n$ th-order cosine and sine charge

\footnotetext{
*ken@spring8.or.jp

Published by the American Physical Society under the terms of the Creative Commons Attribution 3.0 License. Further distribution of this work must maintain attribution to the author(s) and the published article's title, journal citation, and DOI.
}

moments are obtained by convolution integrals of charge distribution $\rho(r, \theta)$ with extraction functions $r^{n} \cos n \theta$ and $r^{n} \sin n \theta$ (see the Appendix):

$$
\begin{aligned}
& \int_{0}^{2 \pi} \int_{0}^{\infty} \rho(r, \theta) r^{n} \cos n \theta r d r d \theta, \\
& \int_{0}^{2 \pi} \int_{0}^{\infty} \rho(r, \theta) r^{n} \sin n \theta r d r d \theta .
\end{aligned}
$$

Another theoretical feature of this paper is its consideration of discrete charge distribution, which consists of infinitely small particles instead of continuous distribution [1]. Because a charged particle beam is a system that consists of multiple particles, the higher-order (multipole) moments of a multiparticle beam are considered the sum of the multipole moments of the individual particles. Furthermore, the electric field is considered a superposition of the fields generated by each particle. Therefore, we calculate the multipole moments of each single particle of the beam (single-particle system) and total them to get the multiple moments for the entire beam (multiparticle system). The electric field is computed in a similar way: first for each single particle (single-particle system) and then taking the superposition of the results to acquire the electric field generated by the complete multiparticle distribution (multiparticle system). Applying these procedures, the analysis of multipole moments and multiparticle systems are clearly separated and distinguishable.

In the SPring-8 linac, four-electrode BPMs of two different apertures are currently installed to fit the transverse beam envelope. One has a circular cross section and is used in the nondispersive section of the linac [4]; the other one has a quasielliptical cross section and is used in the dispersive section [5]. A BPM with a quasielliptical cross section ensures that the lowest beam-duct cutoff frequency 
at the BPM is well above the detection frequency of the readout system.

Some of these BPMs will be replaced by six-electrode BPMs to measure the second-order moments of the transverse beam charge distribution. Using six electrodes is sufficient to detect the second-order moments, while the signals of the third-order moments might be too small to detect because the signal strength of the $n$ th-order moment is inversely proportional to the $n$th power of the duct radius.

\section{ANALYSIS OF MULTIPOLE MOMENTS WITH RESPECT TO BEAM CHARGE DISTRIBUTION}

Multipole charge moments are quantities associated with beam charge distribution. In this section we define charge distributions and extract multipole moments from these distributions. Finally, we discuss the relation between second-order moments and beam sizes.

\section{A. Charged particle distribution and multipole moments}

First, we derive the charge moments from the charge distribution of a single-particle system. Because the calculation is performed in a two-dimensional transverse plane, a charged particle is not expressed as a point but as an infinitely long line charge. Assume a point charge with line charge density $\lambda$, located at position $(r, \theta)=(b, \beta)$ using polar coordinates, as shown in Fig. 1.

The single-particle distribution $\rho_{\text {single }}(r, \theta)$ can be expressed by a delta function:

$$
\rho_{\text {single }}(r, \theta)=\frac{\lambda}{\pi r} \delta(r-b) \delta(\theta-\beta) .
$$

The delta function along the $\theta$ axis can be expressed as a series of cosine functions by modifying Eq. (2):

$$
\rho_{\text {single }}(r, \theta)=\frac{\lambda}{\pi r} \delta(r-b)\left[\frac{1}{2}+\sum_{n=1}^{\infty} \cos \{n(\theta-\beta)\}\right] .
$$

Using the definition given by Eqs. (1) and (3), the $n$ thorder cosine and sine charge moments are obtained:

$$
\begin{gathered}
\int_{0}^{2 \pi} \int_{0}^{\infty} \rho_{\text {single }}(r, \theta) r^{n} \cos n \theta r d r d \theta=\lambda b^{n} \cos n \beta=\lambda p_{n}, \\
\int_{0}^{2 \pi} \int_{0}^{\infty} \rho_{\text {single }}(r, \theta) r^{n} \sin n \theta r d r d \theta=\lambda b^{n} \sin n \beta=\lambda q_{n},
\end{gathered}
$$

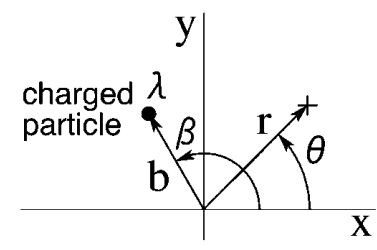

FIG. 1. Configuration of a single-particle system in the twodimensional transverse plane. where

$$
p_{n}=b^{n} \cos n \beta, \quad q_{n}=b^{n} \sin n \beta .
$$

In this paper we define $p_{n}, q_{n}$ as the $n$ th-order cosine and sine moments instead of $n$ th-order cosine and sine charge moments $\lambda p_{n}, \lambda q_{n}$.

Now we derive the $n$ th-order charge moments for a multiparticle system. A multiparticle distribution $\rho_{\text {multi }}(r, \theta)$ can be expressed as a sum of single-particle distributions $\rho_{\text {single } N}(r, \theta)$ s:

$$
\rho_{\text {multi }}(r, \theta)=\sum_{N=1}^{M} \rho_{\text {single } N}(r, \theta) .
$$

In Eq. (6) $N$ is the suffix of the $N$ th particle and $M$ is the number of particles $(M \geqq 2)$. For beam with particles of higher charge states, we consider multiple single charged particles exist at the same transverse position.

Similar to $p_{n}, q_{n}$, we define $n$ th-order cosine and sine moments $P_{n}, Q_{n}$ by convolution integrals of $\rho_{\text {multi }}(r, \theta)$ with corresponding extraction functions $r^{n} \cos n \theta, r^{n} \sin n \theta$,

$$
\begin{aligned}
& \int_{0}^{2 \pi} \int_{0}^{\infty} \rho_{\text {multi }}(r, \theta) r^{n} \cos n \theta r d r d \theta=\lambda \sum_{N=1}^{M} p_{N n}=\Lambda P_{n}, \\
& \int_{0}^{2 \pi} \int_{0}^{\infty} \rho_{\text {multi }}(r, \theta) r^{n} \sin n \theta r d r d \theta=\lambda \sum_{N=1}^{M} q_{N n}=\Lambda Q_{n},
\end{aligned}
$$

where

$$
\begin{aligned}
p_{N n} & =b_{N}^{n} \cos n \beta_{N}, \quad q_{N n}=b_{N}^{n} \sin n \beta_{N}, \quad \Lambda=M \lambda, \\
P_{n} & =a_{n}^{n} \cos n \alpha_{n}=\frac{1}{M} \sum_{N=1}^{M} p_{N n}, \\
Q_{n} & =a_{n}^{n} \sin n \alpha_{n}=\frac{1}{M} \sum_{N=1}^{M} q_{N n}, \\
a_{n}^{n} & =\sqrt{P_{n}^{2}+Q_{n}^{2}}, \quad n \alpha_{n}=\arctan \frac{Q_{n}}{P_{n}}\left(0 \leqq n \alpha_{n}<2 \pi\right) .
\end{aligned}
$$

\section{B. Absolute and relative moments}

A multiparticle system has a center of charge, and the characteristics of this centroid can be expressed by multipole moments.

The previous expressions for $P_{n}, Q_{n}$ are not useful in this context, since we want to know the multipole moments with respect to the centroid of the multiparticle system, not for the origin of the coordinate system. However, since $P_{n}$, $Q_{n}$ are measured quantities using BPMs, we extract relative moments with respect to the centroid from measured absolute moments $P_{n}, Q_{n}$ and the moments of the centroid. To obtain the relative moments we use the following geometric procedure.

Figure 2 shows a multiparticle system. Shown are three particles and their centroids, with the position of the $N$ th particle at $\left(b_{N} \cos \beta_{N}, b_{N} \sin \beta_{N}\right)$. The location of the 


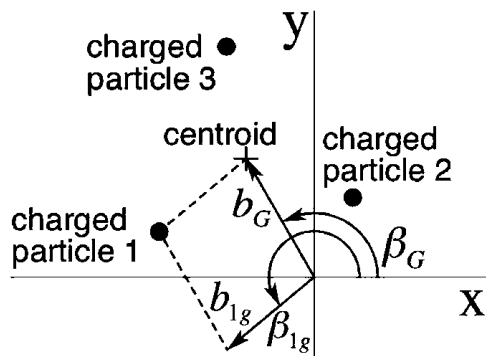

FIG. 2. Example configuration of a typical three-particle system.

centroid is at $\left(b_{G} \cos \beta_{G}, b_{G} \sin \beta_{G}\right)$, and the relative position between the $N$ th particle and the centroid is $\left(b_{N g} \cos \beta_{N g}, b_{N g} \sin \beta_{N g}\right)$, which allows us to express the relative position of the $N$ th particle as

$$
\begin{gathered}
b_{N g} \cos \beta_{N g}=b_{N} \cos \beta_{N}-b_{G} \cos \beta_{G}, \\
b_{N g} \sin \beta_{N g}=b_{N} \sin \beta_{N}-b_{G} \sin \beta_{G} .
\end{gathered}
$$

Using Eq. (9) and the addition and subtraction theorems of the trigonometric function, the $n$ th-order relative moments $p_{N g n}, q_{N g n}$ can be expressed by the absolute moments $p_{N n}, q_{N n}$ and the moments of centroid $p_{G n}, q_{G n}$ :

$$
\begin{aligned}
& p_{N g 1}=p_{N 1}-p_{G 1}, \quad q_{N g 1}=q_{N 1}-q_{G 1}, \\
& p_{N g 2}=p_{N 2}-p_{G 2}-2 b_{G} b_{N g} \cos \left(\beta_{G}+\beta_{N g}\right), \\
& q_{N g 2}=q_{N 2}-q_{G 2}-2 b_{G} b_{N g} \sin \left(\beta_{G}+\beta_{N g}\right), \\
& p_{N g 3}=p_{N 3}-p_{G 3}-3 b_{G}^{2} b_{N g} \cos \left(2 \beta_{G}+\beta_{N g}\right) \\
&-3 b_{G} b_{N g}^{2} \cos \left(\beta_{G}+2 \beta_{N g}\right), \\
& q_{N g 3}= q_{N 3}-q_{G 3}-3 b_{G}^{2} b_{N g} \sin \left(2 \beta_{G}+\beta_{N g}\right) \\
&-3 b_{G} b_{N g}^{2} \sin \left(\beta_{G}+2 \beta_{N g}\right),
\end{aligned}
$$

where

$$
\begin{aligned}
p_{G n} & =b_{G}^{n} \cos n \beta_{G}, \quad q_{G n}=b_{G}^{n} \sin n \beta_{G}, \\
p_{N g n} & =b_{N g}^{n} \cos n \beta_{N g}, \quad q_{N g n}=b_{N g}^{n} \sin n \beta_{N g} .
\end{aligned}
$$

Here, the fourth-order and higher moments are omitted. In Eq. (10), the left side terms are the relative first-order terms. In the right side of Eqs. (11) and (12), the third terms involve the relative first-order terms. These terms consequently vanish in a multiparticle system, as their moments sum to

$$
\sum_{N=1}^{M} p_{N g 1}=0, \quad \sum_{N=1}^{M} q_{N g 1}=0 .
$$

Before summing all moments of the single-particle system, we define the $n$ th-order relative moments of the multiparticle system with respect to centroid $P_{g n}, Q_{g n}$, which resemble absolute moments $P_{n}, Q_{n}$ in Eqs. (8):

$$
\begin{aligned}
P_{g n} & =a_{g n}^{n} \cos n \alpha_{g n}=\frac{1}{M} \sum_{N=1}^{M} p_{N g n}, \\
Q_{g n} & =a_{g n}^{n} \sin n \alpha_{g n}=\frac{1}{M} \sum_{N=1}^{M} q_{N g n}, \\
a_{g n}^{n} & =\sqrt{P_{g n}^{2}+Q_{g n}^{2}}, \\
n \alpha_{g n} & =\arctan \frac{Q_{g n}}{P_{g n}} \quad\left(0 \leqq n \alpha_{g n}<2 \pi\right) .
\end{aligned}
$$

After summing all moments, we finally obtain the relative moments as the following equations that resemble Eqs. (10)-(12):

$$
\begin{gathered}
P_{g 1}=P_{1}-p_{G 1} \equiv 0, \quad Q_{g 1}=Q_{1}-q_{G 1} \equiv 0, \\
P_{g 2}=P_{2}-p_{G 2}, \quad Q_{g 2}=Q_{2}-q_{G 2}, \\
P_{g 3}=P_{3}-p_{G 3}-3 b_{G} a_{g 2}^{2} \cos \left(\beta_{G}+2 \alpha_{g 2}\right), \\
Q_{g 3}=Q_{3}-q_{G 3}-3 b_{G} a_{g 2}^{2} \sin \left(\beta_{G}+2 \alpha_{g 2}\right) .
\end{gathered}
$$

Again, moments of fourth order and higher are not taken. The procedure shows that moments of third order and higher cannot be simply expressed solely by the moments. Cross products appear, however, if the centroid is located at the origin of the coordinate system, i.e., $b_{G}=0$; these cross terms vanish and the relative moments equal the absolute moments, e.g., $P_{g n}=P_{n}, Q_{g n}=Q_{n}$.

\section{Second-order relative moments and beam sizes}

Now we discuss the relation between second-order relative moments and transverse beam sizes. First, we define axes $u, v$ rotated by $\alpha_{g 2}$ from axes $x, y$ (Fig. 3).

For an $N$ th particle, argument $\gamma_{N g}$ can be defined as

$$
\gamma_{N g}=\beta_{N g}-\alpha_{g 2}
$$

Using Eqs. (15) and (19) we obtain the following system of identities:

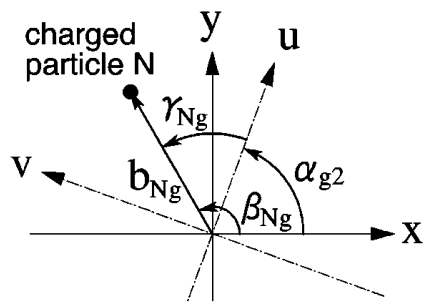

FIG. 3. Definition of rotated axes $u, v$. 


$$
\begin{aligned}
a_{g 2}^{2} \cos 2 \alpha_{g 2} \equiv & \frac{1}{M} \sum_{N=1}^{M} b_{N g}^{2} \cos 2 \beta_{N g} \\
\equiv & \frac{\cos 2 \alpha_{g 2}}{M} \sum_{N=1}^{M} b_{N g}^{2} \cos 2 \gamma_{N g} \\
& -\frac{\sin 2 \alpha_{g 2}}{M} \sum_{N=1}^{M} b_{N g}^{2} \sin 2 \gamma_{N g} \\
a_{g 2}^{2} \sin 2 \alpha_{g 2} \equiv & \frac{1}{M} \sum_{N=1}^{M} b_{N g}^{2} \sin 2 \beta_{N g} \\
\equiv & \frac{\sin 2 \alpha_{g 2}}{M} \sum_{N=1}^{M} b_{N g}^{2} \cos 2 \gamma_{N g} \\
& +\frac{\cos 2 \alpha_{g 2}}{M} \sum_{N=1}^{M} b_{N g}^{2} \sin 2 \gamma_{N g} .
\end{aligned}
$$

The solution to the system is

$$
\begin{aligned}
a_{g 2}^{2} & =\frac{1}{M} \sum_{N=1}^{M} b_{N g}^{2} \cos 2 \gamma_{N g} \\
& =\frac{1}{M} \sum_{N=1}^{M}\left(u_{N g}^{2}-v_{N g}^{2}\right) \\
& =\left\langle u_{g}^{2}\right\rangle-\left\langle v_{g}^{2}\right\rangle \sim \sigma_{u}^{2}-\sigma_{v}^{2}, \\
0 & =\frac{1}{M} \sum_{N=1}^{M} b_{N g}^{2} \sin 2 \gamma_{N g} \\
& =\frac{1}{M} \sum_{N=1}^{M} 2 u_{N g} v_{N g}=2\left\langle u_{g} v_{g}\right\rangle,
\end{aligned}
$$

where

$$
u_{N g}=b_{N g} \cos \gamma_{N g}, \quad v_{N g}=b_{N g} \sin \gamma_{N g} .
$$

This solution means that the beam charge distribution, which only has second-order relative moments, is an ellipse with a long radius along the $u$ axis and a short radius along the $v$ axis. Consequently, $a_{g 2}^{2}$ is expressed as the difference of the squares of beam sizes $\sigma_{u}$ and $\sigma_{v}$.
Figure 4 shows three examples with equivalent secondorder relative moments whose charge distributions are expressed by contour lines. This comparison suggests that different charge distributions can result in identical properties $a_{g 2}$ and $\alpha_{g 2}$ of the second-order relative moments. However, emittances and Twiss parameters can be deduced using more than six $a_{g 2} \mathrm{~s}$ and $\alpha_{g_{2}} \mathrm{~s}$ in combination with a focusing-defocusing (FODO) magnetic array [6].

\section{ANALYTICAL ANALYSIS OF A SIX-ELECTRODE BPM WITH CIRCULAR CROSS SECTION}

In this section we derive an analytical formulation that describes the relation between the multipole absolute moments and the electrode output signals of a six-electrode BPM with circular cross section. Before, we briefly review the electric field inside a metallic duct with circular cross section.

\section{A. Analysis of electric field inside metallic duct with circular cross section}

Again, assume a point charge is located at $(r, \theta)=$ $(b, \beta)$ in the polar coordinates inside a metallic duct with a circular cross section. The origin is the center of the duct (Fig. 5).

The two-dimensional electrostatic potential $\phi(r, \theta)$ of this configuration is evaluated by applying a method of images, using a mirror point charge [7]:

$$
\begin{aligned}
\phi(r, \theta)= & \frac{\lambda}{2 \pi \varepsilon_{0}}\left\{\ln \frac{1}{\sqrt{r^{2}+b^{2}-2 r b \cos (\theta-\beta)}}\right. \\
& \left.-\ln \frac{1}{\sqrt{r^{2}+\left(\frac{R^{2}}{b}\right)^{2}-\frac{2 r R^{2}}{b} \cos (\theta-\beta)}}+\ln \frac{b}{R}\right\} .
\end{aligned}
$$

Term $\ln (b / R)$ is a constant to ground the metallic duct. The first term of the right-hand side is generated by the charged particle, and the second term is generated by the mirror particle. Using the addition and subtraction theorems of the trigonometric functions, Eq. (23) can be approximately expanded to the Taylor series under assumption $b \ll r \sim R$ :
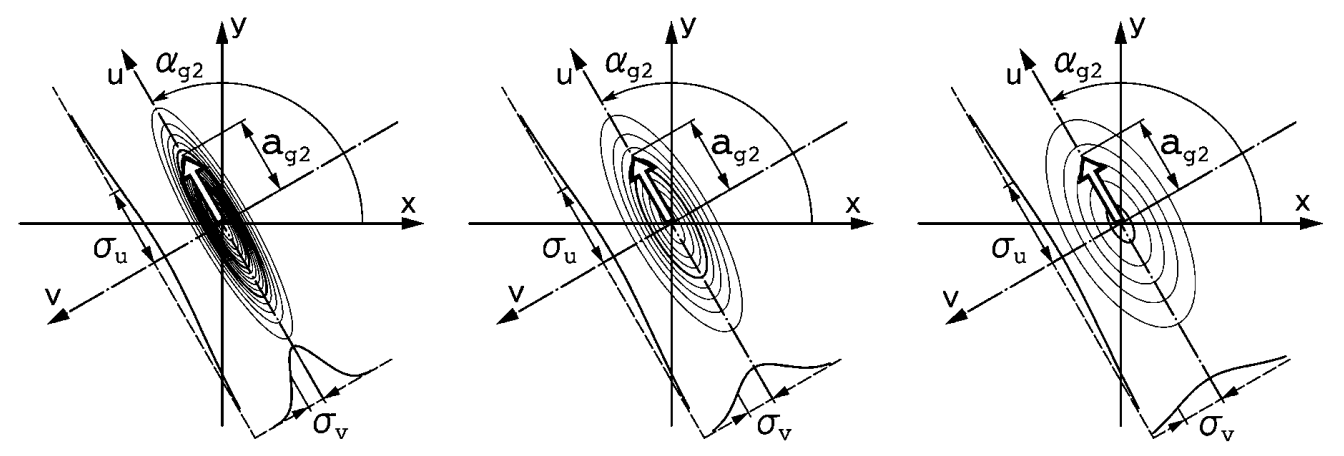

FIG. 4. Three examples of beam charge distributions with equivalent second-order relative moments when $a_{g 2}=7$ and $\alpha_{g 2}=$ $2 \pi / 3$. Three parameters of $\left(\sigma_{u}, \sigma_{v}\right)$ are $(7.16,1.49),(7.43,2.48)$, and $(7.96,3.78)$. 


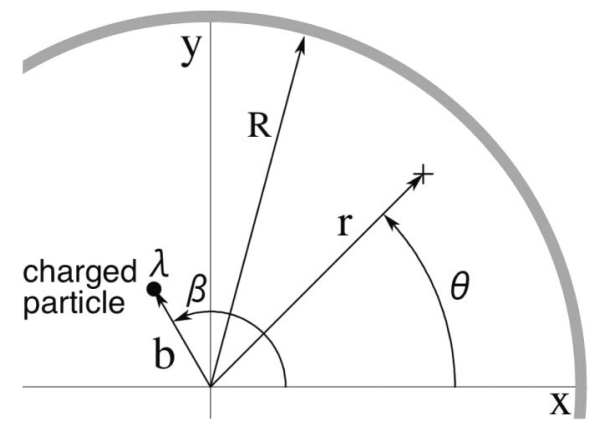

FIG. 5. Configuration of a single point charge in a metallic duct with circular cross section in the $2 \mathrm{D}$ transverse plane (polar coordinates).

$$
\phi(r, \theta)=\frac{\lambda}{2 \pi \varepsilon_{0}}\left[\ln \frac{R}{r}+\sum_{n=1}^{\infty} \frac{b^{n}}{n}\left(\frac{1}{r^{n}}-\frac{r^{n}}{R^{2 n}}\right) \cos \{n(\theta-\beta)\}\right] .
$$

The electric field components are given as the differential of Eq. (24):

$$
\begin{aligned}
& E_{r}(r, \theta)=\frac{\lambda}{2 \pi \varepsilon_{0}}\left[\frac{1}{r}+\sum_{n=1}^{\infty} b^{n}\left(\frac{1}{r^{n+1}}+\frac{r^{n-1}}{R^{2 n}}\right) \cos \{n(\theta-\beta)\}\right], \\
& E_{\theta}(r, \theta)=\frac{\lambda}{2 \pi \varepsilon_{0}} \sum_{n=1}^{\infty} b^{n}\left(\frac{1}{r^{n+1}}-\frac{r^{n-1}}{R^{2 n}}\right) \sin \{n(\theta-\beta)\} .
\end{aligned}
$$

Because we are interested in the field at the inner surface of the duct, $R$ is substituted for $r$ in Eqs. (24) and (25):

$$
\phi(R, \theta)=0
$$

$E_{r}(R, \theta)=\frac{\lambda}{2 \pi R \varepsilon_{0}}\left\{1+2 \sum_{n=1}^{\infty} \frac{p_{n} \cos n \theta+q_{n} \sin n \theta}{R^{n}}\right\}$,

$E_{\theta}(R, \theta)=0$.

Consequently, the potential and angular components of the electric field vanish at the inner surface of the duct.

For a multiparticle system, electric field $E_{M r}(R, \theta)$ is expressed as a superposition of $E_{N r}(R, \theta) \mathrm{s}$ in Eq. (27):

$$
\begin{aligned}
E_{M r}(R, \theta) & =\sum_{N=1}^{M} E_{N r}(R, \theta) \\
& =\frac{\lambda}{2 \pi R \varepsilon_{0}} \sum_{N=1}^{M}\left\{1+2 \sum_{n=1}^{\infty} \frac{p_{N n} \cos n \theta+q_{N n} \sin n \theta}{R^{n}}\right\} \\
& =\frac{\Lambda}{2 \pi R \varepsilon_{0}}\left\{1+2 \sum_{n=1}^{\infty} \frac{P_{n} \cos n \theta+Q_{n} \sin n \theta}{R^{n}}\right\}
\end{aligned}
$$

\section{B. Derivation of multipole moments from BPM outputs}

To derive multipole absolute moments from the BPM electrode signals, we apply a known method that calculates the signal differences between symmetrically arranged BPM electrodes. The choice which differences to use is

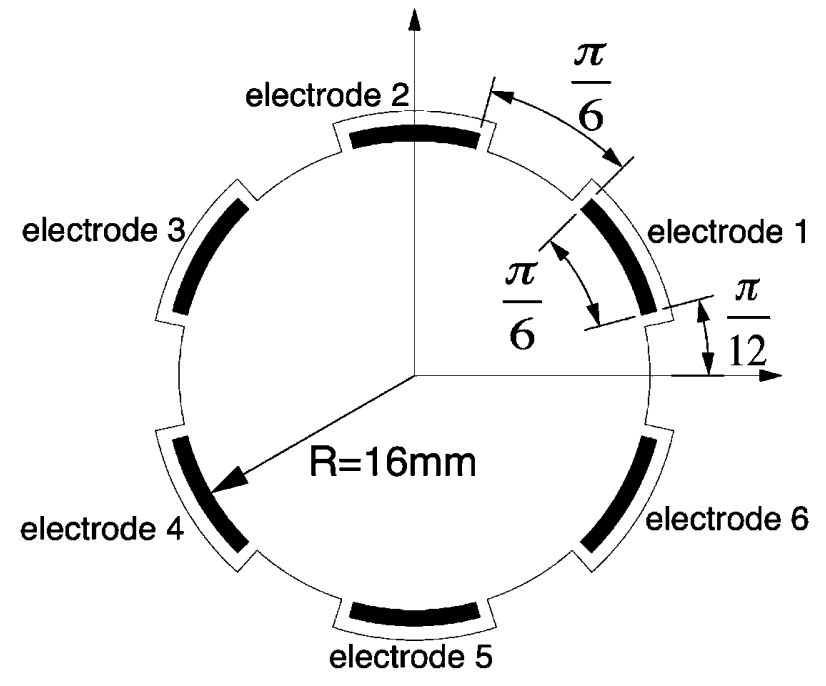

FIG. 6. Configuration of a specific six-electrode BPM with circular cross section.

not arbitrary, therefore we have to find those that return the best measurement resolution. The selected difference has to be sensitive to the specific components of a particular order of that moment but insensitive to other parameters (orthogonality). Since we are interested in second-order moments, we limit our analysis to second orders from now on.

The configuration of the model BPM with a circular cross section is shown in Fig. 6. The number of electrodes is six, and the duct radius is $16 \mathrm{~mm}$. Their shared radius is $\pi / 6$, and the center of the electrodes is located at $(2 d-1) \pi / 6(d=1, \ldots, 6)$ in an angular direction.

The output signals of the BPM electrodes are proportional to the surface integral of the electric field in Eq. (28) on each electrode:

$$
V_{d} \propto R \int_{\{(4 d-3) \pi\} / 12}^{\{(4 d-1) \pi\} / 12} E_{M r}(R, \theta) d \theta, \quad(d=1, \ldots, 6) .
$$

From the integral of formula (29), we obtain each output of electrode $V_{d}$ with geometrical factors $c_{d n}$ and $s_{d n}$ as follows:

$$
V_{d} \propto \frac{\pi}{12}+\sum_{n=1}^{2} \frac{c_{d n} P_{n}+s_{d n} Q_{n}}{R^{n}},
$$

where

$$
\begin{aligned}
c_{d n} & =\int_{\{(4 d-3) \pi\} / 12}^{\{(4 d-1) \pi\} / 12} \cos n \theta d \theta, \\
s_{d n}= & \int_{\{(4 d-3) \pi\} / 12}^{\{(4 d-1) \pi\} / 12} \sin n \theta d \theta, \quad(d=1, \ldots, 6) .
\end{aligned}
$$

The geometrical factors can be expressed using the following four coefficients $f_{1}, h_{1}, f_{2}$, and $h_{2}$ from the integrals of Eqs. (31): 


$$
\begin{aligned}
f_{1} & =c_{11}=-c_{31}=-c_{41}=c_{61}, \\
h_{1} & =s_{11}=s_{31}=-s_{41}=-s_{61}, \\
0 & =c_{21}=c_{51}, \\
2 h_{1} & =s_{21}=-s_{51}, \\
f_{2} & =c_{12}=c_{32}=c_{42}=c_{62}, \\
h_{2} & =s_{12}=-s_{32}=s_{42}=-s_{62}, \\
2 f_{2} & =-c_{22}=-c_{52}, \\
0 & =s_{22}=s_{52} .
\end{aligned}
$$

With the help of formulas (30) and (32) we chose signal differences $C_{n}$ and $S_{n}$, which provide the best resolution for our measurement:

$$
\begin{aligned}
C_{1} & =\frac{V_{1}-V_{3}-V_{4}+V_{6}}{V_{1}+V_{3}+V_{4}+V_{6}}=\frac{12 R f_{1} P_{1}}{\pi R^{2}+12 f_{2} P_{2}} \approx \frac{12 f_{1}}{\pi R} P_{1}, \\
S_{1} & =\frac{V_{2}-V_{5}}{V_{2}+V_{5}}=\frac{24 h_{1}}{\pi R} Q_{1}, \\
C_{2} & =\frac{V_{1}+V_{3}+V_{4}+V_{6}-2\left(V_{2}+V_{5}\right)}{V_{1}+V_{3}+V_{4}+V_{6}+2\left(V_{2}+V_{5}\right)}=\frac{18 f_{2} P_{2}}{\pi R^{2}-6 f_{2} P_{2}} \\
& \approx \frac{18 f_{2}}{\pi R^{2}} P_{2}, \\
S_{2} & =\frac{V_{1}-V_{3}+V_{4}-V_{6}}{V_{1}+V_{3}+V_{4}+V_{6}}=\frac{12 h_{2} Q_{2}}{\pi R^{2}+12 f_{2} P_{2}} \approx \frac{12 h_{2}}{\pi R^{2}} Q_{2} .
\end{aligned}
$$

The approximated results of Eqs. (33) allow us to define ratios $P_{n} / C_{n}$ and $Q_{n} / S_{n}$, which are the normalized moments:

$$
\frac{P_{1}}{C_{1}}=\frac{\pi R}{12 f_{1}}, \frac{Q_{1}}{S_{1}}=\frac{\pi R}{24 h_{1}}, \frac{P_{2}}{C_{2}}=\frac{\pi R^{2}}{18 f_{2}}, \frac{Q_{2}}{S_{2}}=\frac{\pi R^{2}}{12 h_{2}} .
$$

Equation (34) shows simple geometric factors between $P_{n}$ and $C_{n}$ as well as $Q_{n}$ and $S_{n}$. The dimension of the first and second-order normalized moment values are in units of $n$th powers of meters (length).

The $n$th root of the normalized moments is larger than the half of the duct radius $R$, as well as for an arbitrary electrode configuration and other signal difference definitions for a metallic circular BPM:

$$
\sqrt[n]{\frac{P_{n}}{C_{n}}} \gtrsim \frac{R}{2}, \quad \sqrt[n]{\frac{Q_{n}}{S_{n}}} \gtrsim \frac{R}{2} .
$$

Formula (35) indicates that the $n$ th-order normalized moments can be roughly estimated as $R^{n} / 2^{n}$.

For certain $P_{n}$ and $Q_{n}$ of the particle distribution, a low value of the normalized moment results in large values for the signal differences and enables us to measure $P_{n}$ and $Q_{n}$ with better resolution. If we define a dimensionless difference measurement accuracy $w$, the following smallest detectable cosine and sine components of beam sizes $D_{n}$ and $F_{n}$ are obtained:

$$
D_{n}=\sqrt[n]{\frac{P_{n}}{C_{n}} w}, \quad F_{n}=\sqrt[n]{\frac{Q_{n}}{S_{n}} w}
$$

\section{NUMERICAL ANALYSIS OF A BPM WITH CIRCULAR CROSS SECTION}

A numerical software routine was developed to calculate the two-dimensional electrostatic field generated by a single line charge in a BPM of a circular or elliptical cross section. In a multiparticle system, the electric field is computed as a superposition of the fields generated by individual particles; the method is based on the field calculation of a single-particle system. To confirm the software validity and accuracy, the numerically computed values for the normalized moments were compared with the values of the analytical analysis for the same circular BPM, as described in the previous section.

We applied the well-known finite difference method of successive over-relaxation for the numerical field analysis to solve the Laplace equation in two dimensions. Its software routine uses a square mesh with $50-\mu \mathrm{m}$ distance $\Delta$ between the mesh lines, as shown in Fig. 7. The following was the calculation technique. The single point charge was located at a stationary node to a constant potential, while all the nodes of the metallic duct were set to zero volts (ground). With these initial conditions the electrostatic potential was successively calculated for each node, based on the known finite difference iteration algorithm:

$\Phi_{I, J, K+1}=\frac{\Phi_{I-1, J, K}+\Phi_{I+1, J, K}+\Phi_{I, J-1, K}+\Phi_{I, J+1, K}}{4}$.

In Eq. (37), $I$ and $J$ are the spatial mesh indices and $K$ denotes the iteration index. The electric field $E_{x I J}, E_{y I J}$ was obtained by taking the spatial difference of the potential:

$$
E_{x I J}=\frac{\Phi_{I+1, J}-\Phi_{I-1, J}}{2 \Delta}, \quad E_{y I J}=\frac{\Phi_{I, J+1}-\Phi_{I, J-1}}{2 \Delta} .
$$

To reduce the error of the electric field vectors, grounded nodes were set outside by one or more nodes (Fig. 8),

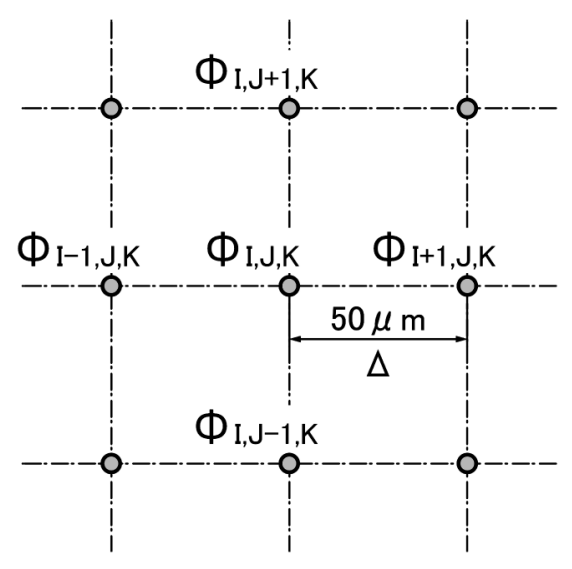

FIG. 7. Nodes for numerical calculation. The mesh was square. 


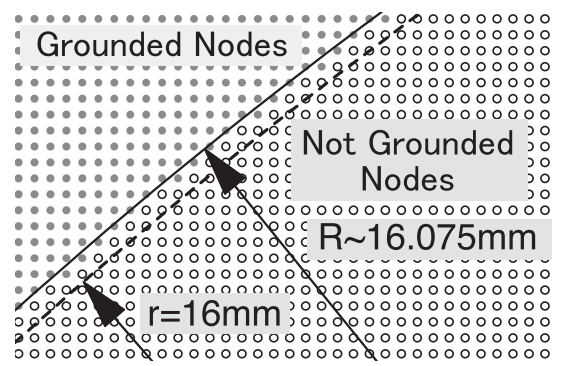

FIG. 8. Grounded nodes set outside by one or more nodes.

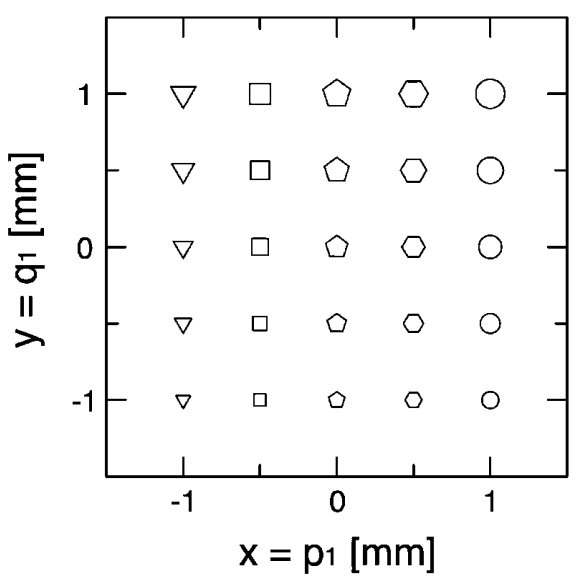

FIG. 9. Location of 25 test charges.
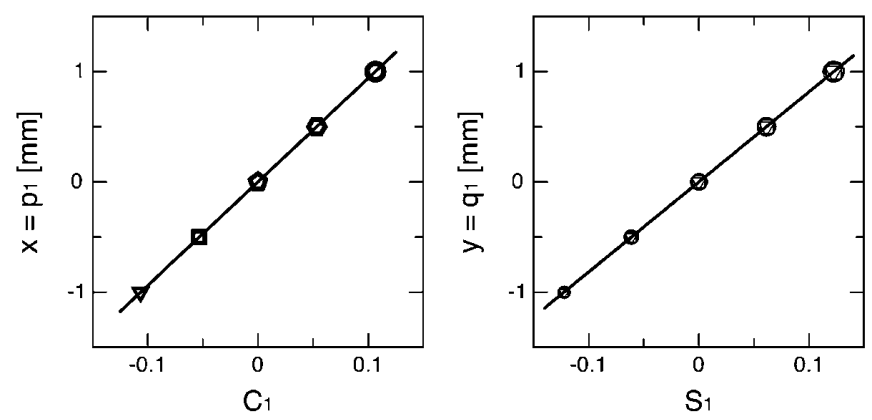

FIG. 10. Relation between $p_{1}$ and $C_{1}, q_{1}$ and $S_{1}$ of the numerically calculated BPM with circular cross section.
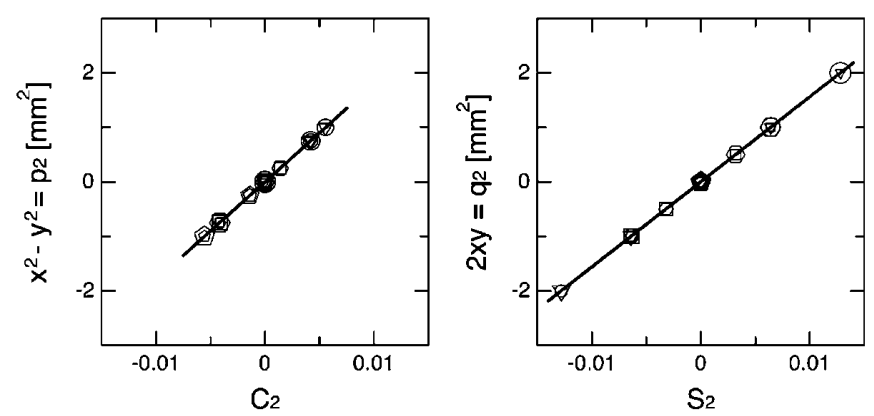

FIG. 11. Relation between $p_{2}$ and $C_{2}, q_{2}$ and $S_{2}$ of the numerically calculated BPM with circular cross section.
TABLE I. Normalized moments: Circular BPM.

\begin{tabular}{lcc}
\hline \hline Normalized moment & Analytically & Numerically \\
\hline$p_{1} / C_{1}$ & 9.346 & 9.394 \\
$q_{1} / S_{1}$ & 8.093 & 8.165 \\
$p_{2} / C_{2}$ & $13.37^{2}$ & $13.42^{2}$ \\
$q_{2} / S_{2}$ & $12.44^{2}$ & $12.50^{2}$ \\
\hline \hline
\end{tabular}

avoiding the neighboring nodes around $r=16 \mathrm{~mm}$ in Eqs. (38) to be grounded. This decreased the $n$ th-order radial field amplitude $b^{n}\left(1 / r^{n+1}+r^{n-1} / R^{2 n}\right)$ in Eq. (25) by $\sim 0.5 \% / n$ because 16 and $16.075 \mathrm{~mm}$ (typically) were substituted for $r$ and $R$. Therefore the numerical calculation of the normalized moments were increased by $\sim 0.5 \% / n$ compared to the analytical results. However, this difference could be reduced by decreasing $\Delta(\Delta \rightarrow 0)$.

Numerical calculations were performed using 25 test charges placed in a symmetric $2 \times 2 \mathrm{~mm}$ square around the origin (Fig. 9).

Figures 10 and 11 show the results of the numerical calculation. The ordinates represent the absolute moments $p_{n}$ and $q_{n}$, the abscissas represent the signal differences $C_{n}$ and $S_{n}$. The normalized moments, which appear as linear coefficients in the figures, are summarized in Table I.

In Table I, the numerically calculated normalized moments are about $0.5 \% / n$ larger than the analytically calculated normalized moments. This phenomenon was predicted in this section. Therefore, it was foreseeable that the numerically calculated normalized moments agree well with the analytically calculated, thus confirmed by comparing the results.

\section{NUMERICAL ANALYSIS OF A BPM WITH ELLIPTICAL CROSS SECTION}

In this section, we analyze a BPM with elliptical cross section using the numerical method. The elliptical BPM with six electrodes and its geometric parameters are shown in Fig. 12. It has similar dimensions as the four-electrode SPring-8 linac BPM with a quasielliptical cross section. The long and short radii are 31 and $15 \mathrm{~mm}$, and the shared angle of the electrodes is $\pi / 6$, and their centers are angularly located at $(2 d-1) \pi / 6(d=1, \ldots, 6)$.

For this configuration we apply the same differences, as discussed in Eq. (33), except for

$$
C_{2} \rightarrow C_{2}^{\prime}=\frac{k\left(V_{1}+V_{3}+V_{4}+V_{6}\right)-2\left(V_{2}+V_{5}\right)}{k\left(V_{1}+V_{3}+V_{4}+V_{6}\right)+2\left(V_{2}+V_{5}\right)}
$$

where $k$ is a constant that corrects $C_{2}^{\prime}$ to zero, if a charged particle is located in the beam-duct center:

$$
k\left(V_{1}+V_{3}+V_{4}+V_{6}\right)-2\left(V_{2}+V_{5}\right)=0,
$$

when a charged particle is located in the beam-duct center. 


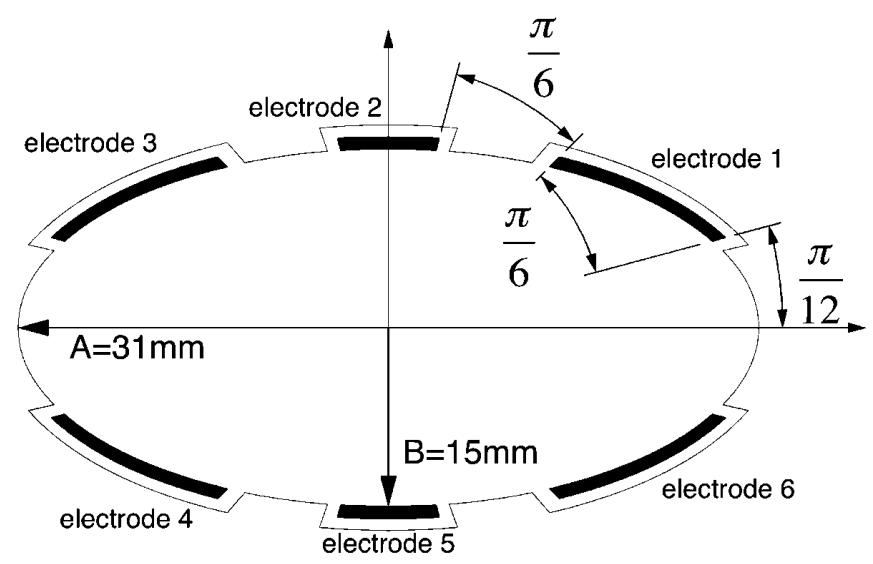

FIG. 12. Configuration of a specific six-electrode BPM with elliptical cross section.
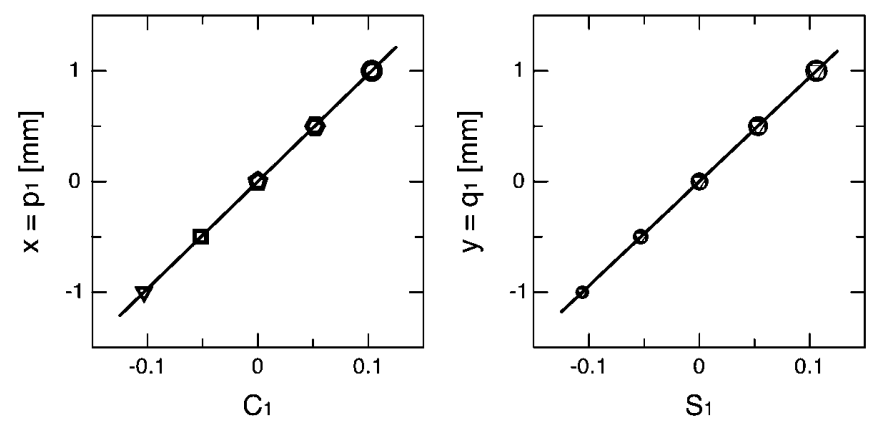

FIG. 13. Relation between $p_{1}$ and $C_{1}, q_{1}$ and $S_{1}$ of the numerically calculated BPM with elliptical cross section.
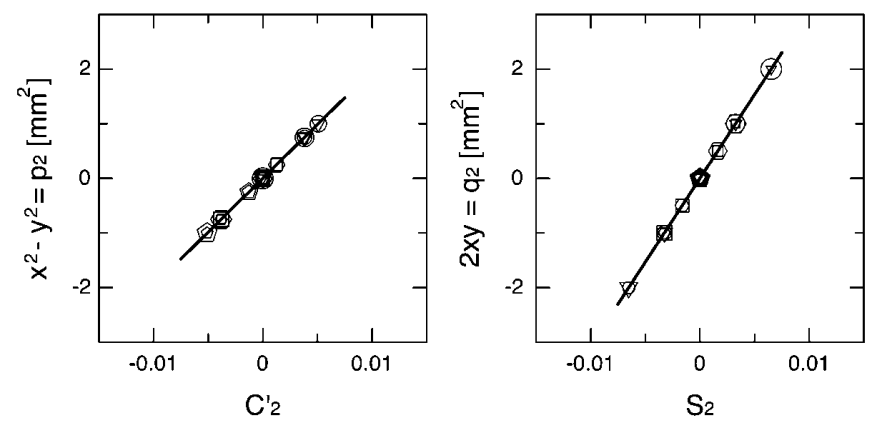

FIG. 14. Relation between $p_{2}$ and $C_{2}^{\prime}, q_{2}$ and $S_{2}$ of the numerically calculated BPM with elliptical cross section.

For this analysis we used the same particle locations, as in the previous circular example (Fig. 9). Figures 13 and 14 show the numerical calculation results. The normalized moments are summarized in Table II; a correction constant of $k=2.162$ was used in Eq. (39).

Comparing the results of the BPMs with circular and elliptical cross section, the normalized moments are larger in the elliptical case. This makes sense, because the chosen elliptical aperture (average beam-duct radius) is larger than the circular.
TABLE II. Normalized moments: Elliptical BPM.

\begin{tabular}{lc}
\hline \hline Normalized moment & Numerically \\
\hline$p_{1} / C_{1}$ & 9.691 \\
$q_{1} / S_{1}$ & 9.417 \\
$p_{2} / C_{2}$ & $14.02^{2}$ \\
$q_{2} / S_{2}$ & $17.52^{2}$ \\
\hline \hline
\end{tabular}

\section{DISCUSSION}

Finally, we discuss the practical applications of the BPM configuration for the measurements of second-order moments in the SPring- 8 linac. The measurement accuracy of the difference signals, which is limited by the noise level of the signal processing electronics, is estimated as $w \sim 1 \times 10^{-3}$ [4]. For this value, based on the results in Table I, we conclude from Eq. (36) that the smallest detectable cosine and sine components of the beam sizes are $D_{1} \sim 9.4 \mu \mathrm{m}, \quad F_{1} \sim 8.2 \mu \mathrm{m}, D_{2} \sim 420 \mu \mathrm{m}$, and $F_{2} \sim 400 \mu \mathrm{m}$ for a BPM with a circular cross section.

The emittance of the SPring- 8 linac is $\sim 5 \times$ $10^{-8} \pi \mathrm{mrad}$ at a beam energy of $1 \mathrm{GeV}$ [8]. If the value of the beta function at the BPM location is estimated to be $5 \mathrm{~m}$, the beam size is $500 \mu \mathrm{m}$. In areas of lower beam energy along the linac, i.e., $<1 \mathrm{GeV}$, the beam size consequently exceeds $500 \mu \mathrm{m}$. This means the second-order moments can be measured in all locations along the linac.

For a BPM with an elliptical cross section used in the dispersive section, the smallest detectable cosine and sine components of the beam sizes are $D_{1} \sim 9.7 \mu \mathrm{m}$, $F_{1} \sim 9.4 \mu \mathrm{m}, D_{2} \sim 440 \mu \mathrm{m}$, and $F_{2} \sim 550 \mu \mathrm{m}$ with the results shown in Table II.

The BPMs with an elliptical cross section, located in the dispersive sections of the linac, are also used for the measurement of the energy spread of the beam. The beam's energy spread was measured to be $\sim 0.15 \%$ (half width at half maximum) behind the energy compression system [8]. The nominal dispersion at these BPM locations is around $0.5 \mathrm{~m}$, and thus the beam size is estimated to be $750 \mu \mathrm{m}$, which also allows us to use these BPM locations for a measurement of second-order moments.

\section{CONCLUSIONS}

We presented a comprehensive theoretical discussion of the multipole moments of a charged particle beam in twodimensional polar coordinates. The charge distribution was considered as a discrete distribution that consists of infinitely small particles instead of a continuous distribution. The multipole moments of the beam were evaluated as the sum of the multipole moments of the charged particles. The electric field generated by the beam was evaluated as the superposition of the electric fields generated by individual particles.

This paper also provides the expressions for the $n$ thorder absolute cosine and sine moments $P_{n}, Q_{n}$ that were 
orthogonal in a two-dimensional plane. The absolute moments were measured quantities using a beam position monitor (BPM). The relative moments with respect to the centroid of beam $P_{g n}, Q_{g n}$, which we were interested in, were presented using the moments of the centroid $p_{G n}$, $q_{G n}$. Second-order relative moments $P_{g 2}, Q_{g 2}$ were associated with the beam sizes of long and short radii $\sigma_{u}, \sigma_{v}$ of the elliptical beam shape.

We defined normalized moments $P_{n} / C_{n}, Q_{n} / S_{n}$, which are important parameters to estimate the smallest detectable cosine and sine components of beam sizes $D_{n}, F_{n}$.

Metallic BPMs with a symmetric arrangement of six electrodes of circular, as well as elliptical cross sections were scrutinized. A numerical analysis, solving the twodimensional electrostatic field problem, validated the analytical analysis for a BPM with a circular cross section. For a six-electrode BPM with elliptical cross section, a correction constant $k$ was necessary for accurate measurements of the second-order moments.

We finally discussed the practical limitations due to the measurement accuracy for the SPring- 8 linac. The beam sizes in all areas, including the dispersive sections, are large enough $(\geqq 500 \mu \mathrm{m}$ for nondispersive, $\geqq 750 \mu \mathrm{m}$ for dispersive section) to allow accurate measurements of the second-order moments with six-electrode BPMs with circular and elliptical cross sections.

\section{APPENDIX}

Two-dimensional charge distributions are identically presented in both Cartesian and polar coordinates. They are explicitly expressed as charged particle distributions using the following delta functions:

$$
\begin{aligned}
\rho(x, y) & =\lambda \sum_{N=1}^{M} \delta\left(x-x_{N}\right) \delta\left(y-y_{N}\right), \\
\rho(r, \theta) & =\frac{\lambda}{\pi r} \sum_{N=1}^{M} \delta\left(r-r_{N}\right) \delta\left(\theta-\theta_{N}\right), \\
x_{N} & =r_{N} \cos \theta_{N}, \quad y_{N}=r_{N} \sin \theta_{N},
\end{aligned}
$$

where $\lambda$ is the line charge density, $N$ is the suffix of the $N$ th particle, and $M$ is the number of particles $(M \geqq 2)$. For beam with particles of higher charge states, we consider multiple single charged particles exist at the same transverse position.

Let us define two-dimensional $n$ th-order complex charge moment $L_{n}$ :

$$
\begin{aligned}
L_{n} & =\int_{-\infty}^{\infty} \int_{-\infty}^{\infty} \rho(x, y)(x+i y)^{n} d x d y, \\
& =\int_{0}^{2 \pi} \int_{0}^{\infty} \rho(r, \theta) r^{n} e^{i n \theta} r d r d \theta,
\end{aligned}
$$

where $i=\sqrt{-1}$. The $n$ th-order complex moments are written as follows:

$$
\begin{aligned}
& L_{0}=M \lambda=\Lambda, \\
& L_{1}=m_{10}+i m_{01}=\Lambda P_{1}+i \Lambda Q_{1}, \\
& L_{2}=m_{20}-m_{02}+2 i m_{11}=\Lambda P_{2}+i \Lambda Q_{2}, \\
& L_{3}=m_{30}-3 m_{12}+i\left(3 m_{21}-m_{03}\right)=\Lambda P_{3}+i \Lambda Q_{3}, \\
& L_{4}=m_{40}-6 m_{22}+m_{04}+4 i\left(m_{31}-m_{13}\right)=\Lambda P_{4}+i \Lambda Q_{4},
\end{aligned}
$$

where

$$
\begin{aligned}
m_{j(n-j)} & =\int_{-\infty}^{\infty} \int_{-\infty}^{\infty} \rho(x, y) x^{j} y^{n-j} d x d y, \\
\Lambda P_{n} & =\int_{0}^{2 \pi} \int_{0}^{\infty} \rho(r, \theta) r^{n} \cos n \theta r d r d \theta, \\
\Lambda Q_{n} & =\int_{0}^{2 \pi} \int_{0}^{\infty} \rho(r, \theta) r^{n} \sin n \theta r d r d \theta
\end{aligned}
$$

Here, the fifth-order and higher moments are omitted. Notation $m_{j(n-j)}$ is a traditional expression of the charge moment in Cartesian coordinates [2].

From Eqs. (A3) we obtain the following relations of the charge moments in Cartesian and polar coordinates:

$$
\begin{gathered}
m_{10}=\Lambda P_{1}, \quad m_{01}=\Lambda Q_{1}, \quad m_{20}-m_{02}=\Lambda P_{2}, \\
2 m_{11}=\Lambda Q_{2}, \quad m_{30}-3 m_{12}=\Lambda P_{3}, \quad 3 m_{21}-m_{03}=\Lambda Q_{3}, \\
m_{40}-6 m_{22}+m_{04}=\Lambda P_{4}, \quad 4 m_{31}-4 m_{13}=\Lambda Q_{4} . \quad \text { (A5) }
\end{gathered}
$$

Again, moments of fifth order and higher are not taken.

[1] T. Suwada, Jpn. J. Appl. Phys. 40, 890 (2001).

[2] A. Chapman-Hatchett, A. Jansson, and D. J. Williams, in Proceedings of the 18th Particle Accelerator Conference, New York, 1999 (IEEE, New York, 1999), pp. 2223-2225.

[3] T. Suwada, M. Satoh, and K. Furukawa, Phys. Rev. ST Accel. Beams 6, 032801 (2003).

[4] K. Yanagida, T. Asaka, H. Hanaki, T. Hori, T. Kobayashi, A. Mizuno, S. Suzuki, T. Takashima, and T. Taniuchi, in Proceedings of the 20th International Linac Conference, Monterey, CA, 2000 (SLAC, Menlo Park, CA, 2000), pp. 190-192.

[5] K. Yanagida, T. Asaka, H. Dewa, H. Hanaki, T. Kobayashi, A. Mizuno, S. Suzuki, T. Taniuchi, and H. Tomizawa, in Proceedings of the 22th International Linear Accelerator Conference, Lübeck, Germany, 2004 [http://accelconf.web.cern.ch/accelconf/104/PAPERS/ TUP73.PDF], pp. 438-440.

[6] R.H. Miller, J.E. Clendenin, M. B. James, and J.E. Sheppard, in Proceedings of the 12th International Conference on High-Energy Accelerators (HEAC'83) Fermilab, 1983 (Fermilab, Illinois, 1983), pp. 603-605.

[7] J. D. Jackson, Classical Electrodynamics (John Wiley \& Sons, New York, 1975), 2nd ed., p. 81.

[8] T. Asaka, Y. Kawashima, T. Takashima, T. Kobayashi, T. Ohshima, and H. Hanaki, Nucl. Instrum. Methods Phys. Res., Sect. A 516, 249 (2004). 